\title{
EVALUAR EL DESEMPEÑO EN EL MARCO DEL MODELO EDUCATIVO BASADO EN COMPETENCIAS EN EDUCACIÓN SUPERIOR CON APOYO DE REDES SOCIALES: UN ESTUDIO DE CASO
}

\section{Performance evaluation in competence-based learning model in higher education scenarios using social network: a case study}

\author{
Katherina Edith Gallardo Córdova \\ Tecnológico de Monterrey (México) \\ Correo-e: katherina.gallardo@itesm.mx \\ María Eugenia GiL Rendón \\ Tecnológico de Monterrey (México) \\ Correo-e: maru.gil @itesm.mx \\ Alejandra Laura Govea Garza \\ Tecnológico de Monterrey (México) \\ Correo-e: agovea@itesm.mx \\ Recepción: 23 de septiembre de 2015 \\ Envío a informantes: 31 de octubre de 2015 \\ Aceptación definitiva: 28 de febrero de 2016
}

REsumen: Se presenta una investigación sobre la conducción de un proceso de evaluación de desempeño en un curso de posgrado ofrecido en línea, diseñado bajo el modelo de competencias. Se utilizó la red social Facebook como herramienta interactiva que permitiría compartir información en formato de video con la intención de ilustrar tanto aspectos de los diversos contextos educativos como los impactos de la ejecución de los proyectos de mejora, en opinión de los diferentes beneficiarios. Para el estudio se optó por una metodología cualitativa bajo el enfoque estudio de caso. Se seleccionaron 7 unidades de análisis de 34 posibles. Los casos se documentaron en escenarios escolares de México y Colombia luego de la implementación de un proyecto de mejora en materia de evaluación del aprendizaje. Se integraron una serie de evidencias del desempeño de los estudiantes a partir de los productos generados. Los hallazgos permitieron corroborar que los estudiantes trabajaron en sus contextos de manera alineada con las competencias académicas estipuladas. Además, a través de las evidencias como el material compartido 
en Facebook, se pudo conocer los beneficios derivados de los proyectos para cada una de las comunidades educativas. Se sugiere el uso de redes sociales para fortalecer la evaluación del desempeño que derive en mejoras curriculares y de diseño instruccional.

Palabras Clave: modelo educativo basado en competencias; evaluación del aprendizaje; desempeño; redes sociales.

Aвstract: A research about performance evaluation was conducted in a graduate online course designed in the Based-Competency Model. Facebook was used as a social and interactive tool that would permit sharing information to illustrate various aspects of diverse educational contexts as well as the impacts of the implementation of improvement projects seen from the beneficiaries' perspective. Case Study was the methodology selected. Postgraduate students got the task to work on certain improvements on learning assessment matters. The educational scenarios were located in Mexico and Colombia. 7 units of analysis were chosen among 34 possible. The findings pointed out that students worked on their contexts in alignment with the stipulated academic competencies. The use of video materials posted and shared using Facebook allowed get a deeper understanding of the way the benefits influenced in each of the educational communities. Besides, these products evidenced students' appropriate performance. In conclusion, the use of social networks for fortifying performance assessment is highly recommended. Moreover, it is expected that these benefits also influence some of the curricular and instructional design aspects.

KeY wORDs: Competency based educational model; learning assessment; performance; social networks.

\section{Introducción}

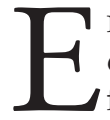
N LOS ÚlTimos io años LA EDUCACión SUPERIOR en América Latina se ha visto enriquecida por la inserción de una serie de innovaciones en el aula, llamadas a fortalecer los ambientes de aprendizaje. Entre las más importantes se encuentra, por un lado, la consolidación del modelo educativo basado en competencias (MEBC) (Coll, 2007; Proyecto Tuning América Latina, 2007), modelo que se catapultó a partir de la obra de Delors et al. (I997) como una respuesta a las necesidades del siglo xxI. En paralelo, el uso de las Tic y la inserción de las redes sociales han incrementado su presencia en diferentes ámbitos, incluido el educativo.

En América Latina se cuenta con 240 millones de usuarios de Internet registrados hasta el 20I5. Una proyección apunta a que, entre el 2013 y el 20I8, se experimentará un incremento de roo millones de usuarios (The Statistical Portal, 20I5). En cuanto al ámbito educativo, Twitter, Facebook, YouTube, LinkedIn y Google Drive están marcando tendencia en cuanto a nuevas formas de participar y colaborar en cursos de pregrado y posgrado (Buzzetto-More, 20I4; Evans, 20I4; García, 2008). Estas estadísticas son muestra del uso cada vez más cotidiano de TIC con diferentes fines educativos en educación superior, donde el MEBC no es la excepción. El problema es que, en la mayor parte de las ocasiones, estas nuevas tecnologías ingresan al proceso enseñanza-aprendizaje sin estrategias planeadas ni consensos formales que coadyuven a elevar la calidad educativa (Earl, 2008; Venkatesh, Croteau y Rabah, 20I4). 
EVALUAR EL DESEMPEÑO EN EL MARCO DEL MODELO EDUCATIVO BASADO EN COMPETENCIAS EN EDUCACIÓN SUPERIOR CON APOYO...

K. E. GALLARDO CÓRDOVA, M. ${ }^{\mathrm{a}}$ E. GIL RENDÓN Y A. L. GOVEA GARZA

Por lo anterior, este artículo aborda el estudio de una estrategia educativa enfocada en mejorar la evaluación del desempeño como el tipo de evaluación que el MEBC demanda. Se partió de la premisa siguiente: la evaluación del desempeño debe guardar congruencia y, por ende, estimar las capacidades de la persona competente en su ámbito de acción, integrando en su actuar elementos cognitivos, afectivos y sociales para responder a necesidades de su entorno (Hancock, 2007; Morrow et al., 20I6; Tobón, 2008; Ministerio de Educación y Ciencia, 2007). Por lo tanto, la integración de diferentes evidencias de desempeño, más allá de las que permitan medir las capacidades individuales, es necesaria.

De allí que considerar diferentes elementos, entre ellos el contexto, podría enriquecer y diversificar las fuentes de donde se obtiene la información para inferir los niveles de competencia alcanzados. Además, permite tener una idea del impacto de las acciones generadas a través de los proyectos emprendidos por los estudiantes a favor del entorno.

\section{Marco teórico}

\section{I. Educación basada en competencias}

A pesar de la importancia que el MEBC ha cobrado en los últimos años, existe cierta controversia por falta de claridad en cuanto a su aplicación en el aula. Gonzi (200I) destaca su aceptación por parte de los sectores industrial y gubernamental en paralelo con el rechazo de quienes califican el $\mathrm{MEBC}$ como rígido, costoso y difícil de comprender.

Tobón (2008) afirma que el MEBC no es un modelo pedagógico sino un enfoque compatible con la propuesta pedagógica de las instituciones educativas. Se debe considerar como un modelo que orienta para el diseño curricular, los procesos de instrucción y las decisiones en torno a la evaluación, todo esto en un marco de calidad basado en principios e indicadores. Cada institución educativa debe partir de su filosofía respecto a qué tipo de persona desean formar, fundamentándola desde su enfoque pedagógico, referentes legales y contexto cultural. Además, se debe tomar el perfil profesional como referencia para orientar la formación hacia la práctica.

Sin duda el мевс se ha ido diversificando. Se pueden distinguir varios enfoques: el conductual, el funcionalista, el constructivista y el sistémico-complejo. Esta diversidad responde a la influencia de distintas disciplinas y demandas socioeconómicas que matizan las concepciones de competencia. Para fines de este estudio se favoreció trabajar bajo el enfoque sistémico-complejo por su compatibilidad con el modelo pedagógico de la institución en la que se realizó esta investigación. Desde esta perspectiva, las competencias se definen como procesos complejos de desempeño con idoneidad y ética ante actividades y problemas, buscando la realización personal, la calidad de vida y el desarrollo social y económico sostenible y en equilibro con el ambiente (Tobón, 2008). Los procesos de instrucción se focalizan en el desempeño integral de las personas y en la contextualización de los saberes para lograr así una articulación del saber conocer, saber ser, saber hacer y saber convivir propuestos por Delors et al. (1997).

El мЕвс, en sus diferentes variantes, reta a la comunidad educativa a trabajar en un proceso conjunto de toma de decisiones y emisión de soluciones para dar respuestas 
apropiadas en su contexto (Alarcón, Hill y Frites, 20I4; Argudin, 20Io). Es momento que las instituciones, en especial las de nivel superior, tomen una postura y decisiones en relación con la instauración de este modelo para responder a demandas económicas y sociales.

\subsection{Evaluación en el marco del modelo educativo basado en competencias}

Por la naturaleza del enfoque, la evaluación del desempeño en el MEBC es un proceso que se integra desde la etapa de planeación. El hecho de privilegiar el desempeño del estudiante como manifestación de sus saberes aplicados a diversas situaciones requiere que se definan a priori los alcances, circunstancias y condiciones en los cuales se valorará qué tanto se han llegado a desarrollar las capacidades estipuladas (Gallardo Córdova, 2007; Lozano-Rodriguez y Herrera-Bernal, 20I2; Pavié, 20II).

El estado del arte marca que uno de los aspectos más urgentes a atender en el MEBC gira en torno al abordaje de la evaluación del desempeño en escenarios de educación superior. Ashford-Rowe et al. (2014) y Monereo (2007) afirman que es preciso que los ambientes educativos que se distinguen por trabajar en el MEBC aseguren las condiciones de evaluación auténtica, es decir, que se planeen y ejecuten actividades que se acerquen lo más posible a los escenarios reales donde ejercerá al terminar sus estudios. Esta afirmación se vincula con lo que Tovar-Gálvez, García et al. (20I2) postulan: el marco contextual es desde donde se conciben e interpretan las competencias para luego evaluarse. Así, el factor contextual cobra valor en tanto la observación y la determinación, medición y recolección de evidencias permitan estimar los alcances del aprendizaje logrado. Por lo anterior, señalan «la evaluación de competencias requiere tiempo suficiente para confrontar al sujeto con su realidad y tomar evidencias de criterios múltiples que respondan a dicha realidad» (p. I26).

En cuanto a las competencias docentes necesarias para llevar estos consejos a la práctica, Ion y Cano (20II) realizaron un estudio con cinco instituciones españolas obligadas a trabajar con el МЕвс por los acuerdos del tratado de Bolonia. Se concluyó que, aunque el interés de los docentes por conocer más sobre el modelo y los procesos de evaluación es una constante, se requiere planear y conducir programas de capacitación efectiva. Los autores destacan que los programas actuales se diseñaron con poca vinculación a la esencia del modelo. La fijación por prácticas y actividades evaluativas que obedecen más al paradigma conductista representan un obstáculo.

\subsection{Redes sociales como estrategia educativa}

Sin duda, las redes sociales se han convertido en los últimos años en el medio de comunicación electrónico más concurrido y de mayor crecimiento en el mundo desde el lanzamiento de SixDegrees.com, considerada la primera en su categoría (Boyd y Ellison, 2008). Estos medios masivos, globales e interactivos se estiman de gran utilidad para compartir información en diferentes temáticas y actividades, además de ampliar y fortalecer el capital relacional de las personas. 
EVALUAR EL DESEMPEÑO EN EL MARCO DEL MODELO EDUCATIVO BASADO EN COMPETENCIAS EN EDUCACIÓN SUPERIOR CON APOYO...

K. E. GALLARDO CÓRDOVA, M. ${ }^{\mathrm{a}}$ E. GIL RENDÓN Y A. L. GOVEA GARZA

En relación con las investigaciones recientes realizadas en el tema de uso de redes sociales en contextos educativos, se encuentran agrupadas en: (I) procesos de interacción académica y colaboración (Gómez, Roses y Farias, 20I2; Ponce-Rojo et al., 20I2); (2) preparación y comunicación entre docentes (Túñez y Sixto, 20I2) e, inclusive, (3) prácticas de webmarketing de las universidades (Valerio-Ureña et al., 2015).

Un estudio que coadyuva en la desmitificación de posibles riesgos en el uso de las redes sociales en estudiantes de nivel superior lo abordaron Valerio-Ureña, Leyva et al. (2014). Se midió la capacidad de memorización en relación con la intensidad de uso de las redes sociales; esto a consecuencia de estudios previos sobre una posible relación desfavorable del uso de redes sociales con la vida estudiantil. Se trabajó con 5I estudiantes. Los resultados apuntaron a rechazar la idea de que el uso intensivo de redes sociales estuviera relacionado con dificultades para memorizar contenido disciplinario. Otra investigación que abordó la actividad académica de las redes sociales en el contexto francés en el nivel de posgrado fue presentada por Panckhurst y Marsh (20II). El estudio demandó la colección de datos por un lapso de tres años, analizando cinco casos en torno a ambientes de aprendizaje en línea y uso de redes sociales. En el último caso se hizo un cambio de enfoque hacia la elevación de responsabilidad para los estudiantes, quienes tomaron la iniciativa de delinear objetivos, temas de estudio y formas de trabajo, dejando de lado la imposición temática y metodológica del profesor. Se comprobó que los diseños pedagógicos que privilegian la interacción y la colaboración con uso de redes sociales no ponen en peligro la diversidad, la autonomía, la apertura y la interacción en aprendices adultos, llegando a los objetivos de aprendizaje estipulados.

\section{Método}

Este estudio se realizó bajo el enfoque de estudio de caso para profundizar en el conocimiento sobre el fenómeno educativo y evaluativo. Se optó por el tipo instrumental bajo un modelo incrustado (Merriam, 2009; Yin, 2003). La justificación subyace en que no se tiene información sobre un estudio similar, en el que las redes sociales sean el medio por el cual se puede tener un acceso directo al contexto del aprendiz y que las evidencias de su legado permitan enriquecer los procesos de evaluación del desempeño.

\section{I. Contexto}

El estudio de caso está contenido en un curso denominado Evaluación del aprendizaje (EA), impartido por una universidad privada ubicada al noreste de México que ofrece posgrados en el área de educación, en modalidad en línea, con participación de estudiantes nacionales y extranjeros. En específico, el diseño del curso permitió trabajar alrededor del enriquecimiento y mejora que puede cobrar el proceso de evaluación circunscrito en el MEBC. Las competencias disciplinares y transversales que se tomaron en cuenta para la materia se encuentran en la Tabla I, mismas que se relacionan con el perfil de egreso del programa de posgrado. 
EVALUAR EL DESEMPEÑO EN EL MARCO DEL MODELO EDUCATIVO BASADO EN COMPETENCIAS EN EDUCACIÓN SUPERIOR CON APOYO...

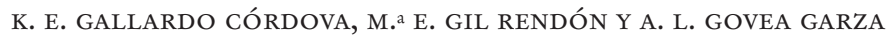

TABLA I: Competencias y subcompetencias disciplinares y transversales del curso Evaluación del aprendizaje

Competencias y
subcompetencias
$\begin{aligned} & \text { disciplinares } \\ & \text { a. Identificar los diferentes momentos del aprendizaje que requieren distintos } \\ & \text { procesos de evaluación del aprendizaje. } \\ & \text { b. Mejorar progresivamente los procesos, mecanismos, emisión y comunicación } \\ & \text { de resultados. } \\ & \text { 2. Diagnosticar las necesidades del contexto educativo: aula, escuela y comunidad. } \\ & \text { a. Indagar en las necesidades educativasy de evaluación del aprendizaje en el } \\ & \text { contexto educativo en donde se desempeña laboralmente o donde se relaciona } \\ & \text { el estudiante de posgrado. } \\ & \text { b. Proponer modelos, formase instrumentación que conlleven enriquecer el } \\ & \text { proceso de diagnóstico del aprendizaje. }\end{aligned}$

3. Detectar e impulsar el talento humano en cada una de las etapas de la evaluación del aprendizaje.

a. Poner a la población a ser evaluada en primer lugar cuando se deben tomar decisiones, respetando en todo momento su calidad de aprendices.

b. Proponer procesos de retroalimentación significativos a favor del logro de las metas educativas trazadas.

4. Conocer tecnologías emergentes y sus usos educativos.
a. Identificar tecnologías emergentes utilizadasen otros países y niveles educativos.
b. Transferir el uso de tecnologías emergentes en contextos específicos hacia situaciones de aprendizaje.

Competencias y

1. Ejercer pensamiento crítico para el análisis de la realidad de su ámbito profesional,

subcompetencias tomando en cuenta puntos de vista variados, conceptos, teorías y explicaciones.

transversales
a. Evaluar los procesos de inferencia que conducen a conclusiones.
b. Emitir juicios de valor acerca de la información.
c. Ser autocrítico al reconocer las propias predisposiciones y su posible sesgo al emitir un juicio de valor.
d. Aceptar la posible desaprobación de otros ante el juicio de valor emitido sobre cierta información.

2. Comunicar información y conocimiento de manera ética a diferentes individuoso grupos utilizando lasTIC.
a. Adecuar la comunicación a las características del contexto y cultura de las audiencias.
b. Comunicarse con otras personas en la búsqueda de acuerdos y posturas comunes sobre problemas compartidos.

EA se ofertó en el semestre agosto-diciembre 2014 por primera vez. Su diseño requirió trabajar dos áreas fundamentales: (I) comprensión teórica y técnica, que sostienen 
EVALUAR EL DESEMPEÑO EN EL MARCO DEL MODELO EDUCATIVO BASADO EN COMPETENCIAS EN EDUCACIÓN SUPERIOR CON APOYO...

K. E. GALLARDO CÓRDOVA, M. ${ }^{a}$ E. GIL RENDÓN Y A. L. GOVEA GARZA

los procesos de evaluación del aprendizaje; y (2) puesta en marcha de un proyecto de intervención para la mejora, iniciando por un diagnóstico, seguido de una planeación y finalmente una intervención con actividades vinculadas a procesos de evaluación formativa y sumativa, considerando ciertos estándares internacionales de evaluación (JCSE, 2OI2), la aplicación de un modelo de retroalimentación (Hattie y Timperley, 2007) y algunas técnicas para llevar diseño y evaluación de pruebas objetivas (Gallardo Córdova, 2013) que derivarían en un manual de procedimientos para la consulta de docentes y directivos. Dentro de las herramientas de interacción se consideró el uso de una plataforma educativa (Blackboard) y una red social ( $\mathrm{Fa}-$ cebook). La Figura i ilustra las actividades en EA, las cuales derivan en la realización de una tarea integradora.

\begin{tabular}{|c|c|c|c|c|c|c|c|c|c|c|c|c|c|c|c|c|}
\hline & \multicolumn{16}{|c|}{ Semanas del semestre } \\
\hline & S1 & S2 & S3 & S4 & S5 & S6 & S7 & 58 & S9 & S10 & S11 & S12 & S13 & S14 & S15 & S16 \\
\hline Unidades & \multicolumn{3}{|c|}{\begin{tabular}{|c|} 
Unidad 1: Retos que \\
enfrenta la evaluación del \\
aprendizaje en el marco \\
de la educación de los \\
ciudadanos del siglo XX
\end{tabular}} & \multicolumn{4}{|c|}{$\begin{array}{l}\text { Unidad 2: Alcances de la evaluación del } \\
\text { aprendizaje }\end{array}$} & \multicolumn{6}{|c|}{$\begin{array}{l}\text { Unidad 3: Planear e instrumentar: tareas cruciales en la } \\
\text { evaluación del aprendizaje }\end{array}$} & \multicolumn{3}{|c|}{$\begin{array}{l}\text { Unidad 4: Comunicar } \\
\text { resultados de la evaluación } \\
\text { del aprendizaje: ir más allá } \\
\text { de emitir una calificación }\end{array}$} \\
\hline \multirow{4}{*}{$\begin{array}{l}\text { Competenci } \\
\text { as } \\
\text { disciplinares }\end{array}$} & \multicolumn{16}{|c|}{ Asumir la evaluación como un proceso formativo del aprendizaje continuo } \\
\hline & \multirow{2}{*}{\multicolumn{7}{|c|}{$\begin{array}{c}\text { Detectar e impulsar el talento humano e } \\
\text { Diagnosticar las necesidades del } \\
\text { contexto educativo: aula, escuela y } \\
\text { comunidad. }\end{array}$}} & en cada & na de $\mathrm{l}$ & etapas de la & la evaluación & $\mathrm{n}$ del apr & dizaje. & & & \\
\hline & & & & & & & & & & & & & & & & \\
\hline & & & & & & & & & & onocertecn & nologias eme & ergentes & sus usos & s educat & & \\
\hline \multirow{2}{*}{\begin{tabular}{|c|}
$\begin{array}{c}\text { Competenci } \\
\text { as } \\
\text { transversale } \\
\text { s }\end{array}$ \\
\end{tabular}} & \multicolumn{16}{|c|}{$\begin{array}{l}\text { Ejercer pensamiento crítico para el análisis de la realidad de su ámbito profesional, tomando en cuenta variados puntos de vista, conceptos, teorias y } \\
\text { explicaciones. }\end{array}$} \\
\hline & \multicolumn{16}{|c|}{ Comunicar información y conocimiento de manera ética a diferentes individuos o grupos utilizando las TIC } \\
\hline $\begin{array}{c}\text { Proyecto: } \\
\text { mejora de } \\
\text { procesos } \\
\text { de } \\
\text { evaluación } \\
\text { del } \\
\text { aprendizaje }\end{array}$ & \multicolumn{5}{|c|}{$\begin{array}{l}\text { Pensar en los } \\
\text { productos: } \\
\text { diagnóstico de } \\
\text { necesidades en los } \\
\text { sistemas de } \\
\text { evaluación del } \\
\text { aprendizaje en las } \\
\text { instituciones }\end{array}$} & $\begin{array}{l}\text { Diseño } \\
\text { mapa de } \\
\text { planeación }\end{array}$ & $\begin{array}{l}\text { Revisión } \\
\text { de la } \\
\text { planeació } \\
\text { n y V.B. } \\
\text { para } \\
\text { continuar }\end{array}$ & \multicolumn{3}{|c|}{$\begin{array}{l}\text { Realización de las diferentes } \\
\text { actividades vinculadas a las } \\
\text { etapas del proyecto Etapa } 1 \text { : } \\
\text { evaluación sumativa }\end{array}$} & $\begin{array}{l}\text { Evaluación } \\
\text { de avances } \\
\text { y } \\
\text { realización } \\
\text { de ajustes }\end{array}$ & $\begin{array}{l}\text { Realiza } \\
\text { diferent } \\
\text { activida } \\
\text { vinculad } \\
\text { etapas } \\
\text { proyect } \\
\text { evaluac } \\
\text { formativ }\end{array}$ & $\begin{array}{l}\text { in de las } \\
\text { s a las } \\
\text { tapa 2: }\end{array}$ & \multicolumn{3}{|c|}{$\begin{array}{l}\text { Tarea integradora: Emisión } \\
\text { de resultados en el reporte } \\
\text { final del proyecto con } \\
\text { emisión de video en página } \\
\text { de Facebook }\end{array}$} \\
\hline
\end{tabular}

FIGURA I: Actividades propuestas para EA a lo largo del semestre

\subsection{Participantes}

Se inscribieron un total de I2I estudiantes, quienes conformaron 34 equipos. Participaron 72 mujeres y 49 varones; sus edades fluctuaron entre 24 y 57 años. Sus países de origen fueron México, Colombia, Costa Rica, Guatemala, Estados Unidos, Ecuador y Perú.

\subsection{Procedimiento}

Selección de elementos del caso a analizar. Con la finalidad de poder concentrar el análisis de elementos representativos del fenómeno se seleccionaron los trabajos de siete equipos de los 34 posibles. Esta selección se hizo con base en algunos criterios: (I) seguimiento a la planeación estipulada, (2) claridad en la expresión de ideas a partir de la grabación con beneficiarios de los proyectos y (3) calidad del audio durante la grabación del video. La Tabla 2 contiene las unidades de análisis seleccionadas. 
EVALUAR EL DESEMPEÑO EN EL MARCO DEL MODELO EDUCATIVO BASADO

EN COMPETENCIAS EN EDUCACIÓN SUPERIOR CON APOYO...

K. E. GALLARDO CÓRDOVA, M. ${ }^{\text {a }}$ E. GIL RENDÓN Y A. L. GOVEA GARZA

TABla 2: Proyectos seleccionados como unidades de análisis

\begin{tabular}{ccccc}
\hline Equipo & País & Ciudad/Región & $\begin{array}{c}\text { Denominación } \\
\text { del escenario } \\
\text { educativo }\end{array}$ & $\begin{array}{c}\text { Nivel educativo al que el proyecto de } \\
\text { evaluación se enfocó }\end{array}$ \\
\hline Equipo 1 & Colombia & Medellín & Escenario 1 & Educación básica (primaria) \\
Equipo 2 & Colombia & Palmar de Varela & Escenario 2 & Educación superior \\
Equipo 3 & México & Morelos & Escenario 3 & Educación básica (secundaria) \\
Equipo 4 & Colombia & Tolima & Escenario 4 & Educación básica (secundaria) \\
Equipo 5 & Colombia & Santander & Escenario 5 & Educación superior \\
Equipo 6 & México & Nuevo León & Escenario 6 & Educación básica (primaria) \\
Equipo 7 & México & Durango & Escenario 7 & Educación básica (secundaria) \\
\hline
\end{tabular}

Los materiales que se analizaron por cada equipo fueron los siguientes: (I) planeación del proyecto de intervención en los temas de evaluación formativa y sumativa; (2) reporte del proyecto integrador; y (3) videos grabados en los cuales se registraron entrevistas con los beneficiarios: profesores y autoridades de las escuelas donde se realizaron los proyectos.

En cuanto al aspecto ético, se solicitaron consentimientos por escrito a cada una de las instituciones y docentes participantes para usos de investigación y divulgación científica. Cada material de video con entrevistas a profesores y directivos fue subido en la página de Facebook del curso por propiciar la discusión, emisión de comentarios y retroalimentaciones a partir de los resultados expresados desde la perspectiva del beneficiario (ver Figura 2).

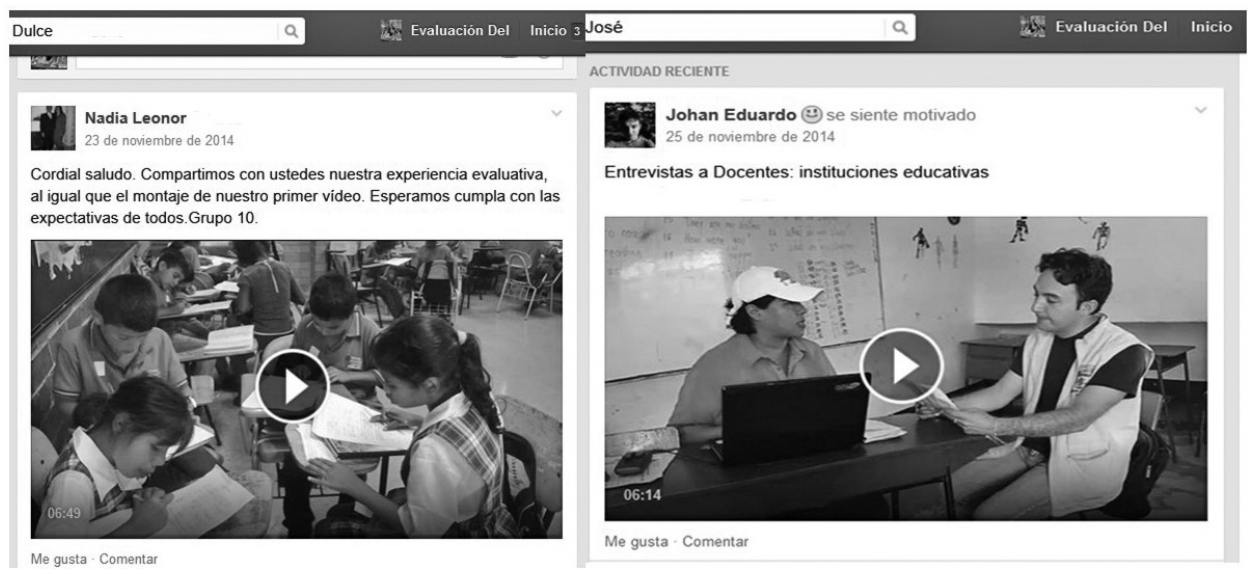

Figura 2: Vista de videos realizados a profesores y directivos publicados en la página de Facebook del curso 
EVALUAR EL DESEMPEÑO EN EL MARCO DEL MODELO EDUCATIVO BASADO EN COMPETENCIAS EN EDUCACIÓN SUPERIOR CON APOYO...

K. E. GALLARDO CÓRDOVA, M. ${ }^{\mathrm{a}}$ E. GIL RENDÓN Y A. L. GOVEA GARZA

\subsection{Tratamiento de los datos}

Merriam (2009) sugiere realizar un proceso de consolidación, reducción e interpretación de lo que otros han dicho y lo que el investigador ha leído y observado. La tarea del procesamiento se vuelve, entonces, un proceso que deriva en hallar patrones dentro de las unidades y organizarlos en un marco coherente para la interpretación. Esta recomendación fue seguida para el análisis en esta investigación, lo cual concluyó en la determinación de temas emergentes durante el proceso de revisión de los videos, escucha de los diálogos y su transcripción.

Así, se escuchó cada entrevista por lo menos tres veces para llegar a un proceso de comprensión más profunda sobre el diálogo y las declaraciones emitidas por los docentes y directivos entrevistados. Luego de la transcripción se introdujeron los archivos en Atlas.ti. Se generaron siete documentos primarios. Posteriormente, se inició con la generación de una lista de códigos, vinculados a familias de códigos que permitieron generar marcos de referencia más amplios. En paralelo se analizaron las planeaciones y reportes para asegurar la coherencia del diálogo con los planes de acción y reportes de resultados.

\section{Resultados}

Los resultados del análisis de los planes de acción, los reportes de los proyectos de los equipos y las entrevistas grabadas y publicadas en Facebook llevaron a identificar cuatro categorías emergentes que explican los resultados de los proyectos de mejora en evaluación educativa que condujeron los estudiantes en los diferentes escenarios: (I) aprendizaje como fin último de la evaluación; (2) innovación educativa para mejorar la evaluación; (3) mejora de la comunicación sobre procesos de evaluación; y (4) mejora de habilidades docentes para evaluar.

Las categorías están relacionadas con competencias y subcompetencias declaradas en el curso (ver Tabla i), las cuales dictan que los estudiantes desarrollarán o perfeccionarán su capacidad de diagnóstico e intervención según las necesidades educativas y de evaluación del aprendizaje en el contexto educativo donde laboran; todo esto con el fin de proponer nuevas o diferentes formas de trabajo para mejorar el proceso. Otra competencia hace referencia a favorecer el sentido humano de la evaluación, poniendo a los estudiantes en primer lugar cuando se tomen decisiones, respetando su calidad de aprendices. Se presenta la Tabla 3, que contiene la cantidad de declaraciones de los beneficiarios (profesores y directivos) alrededor de las cuatro categorías

TABla 3: Frecuencia de aparición de expresiones relacionadas con las cuatro categorías en los siete escenarios educativos

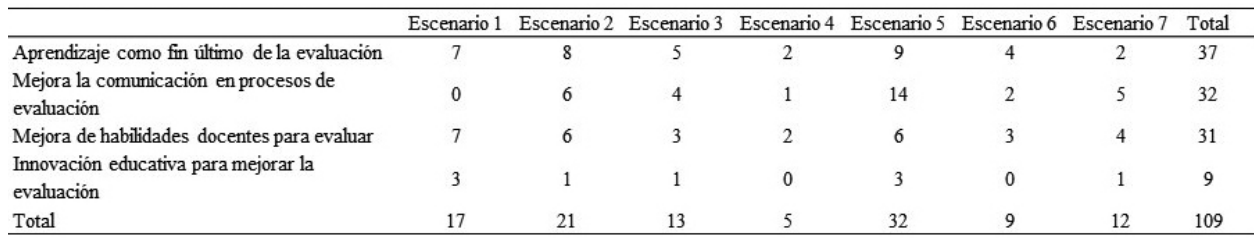


en los diferentes escenarios. Posteriormente, se presentan los resultados cualitativos por categoría.

En los siguientes apartados se presentan resultados sobre la vinculación de los elementos hallados por cada una de las categorías que emergieron del estudio.

\section{I. Aprendizaje como fin último de la evaluación}

En esta primera categoría se hallaron un total de ocho subcategorías relacionadas, de orden conceptual, instrumental y valoral. Se puede inferir que, luego del proceso de trabajo e intervención para la mejora, el compromiso con el aprendizaje emergió como una de las subcategorías más recurrentes, en especial en los escenarios I, 2 y 5 (ver Tabla 3). Otras subcategorías que destacaron fueron las relacionadas con el valor que genera el involucramiento de estudiantes en el proceso de evaluar para aprender (coevaluarse, autoevaluarse), así como la reflexión sobre la valía de la evaluación como un ejercicio para acercarse más a los objetivos de aprendizaje. La Figura 3 muestra los elementos que conforman esta categoría.

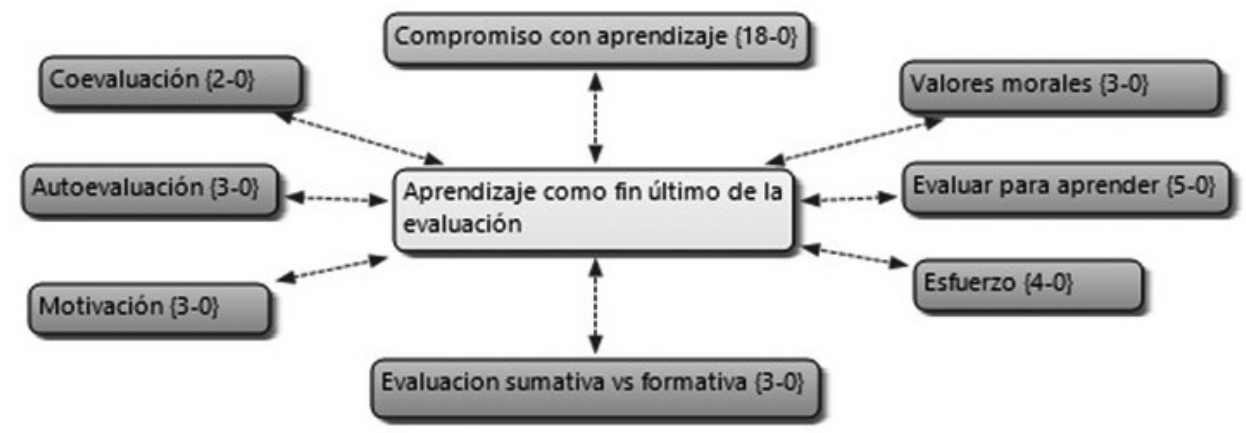

FIGURA 3: Red compuesta por las subcategorías que explican el aprendizaje como fin último de la evaluación

En cuanto a las declaraciones, se resaltan las siguientes:

Profesor I- Escenario 3: relacionado con compromiso con aprendizaje.

[Muchos] retos, eh... que tenemos ahorita en la evaluación es que para todos nuestros alumnos y también a nosotros como docentes la evaluación no se vuelva como un resultado a... a alcanzar sino que sea un... un elemento que nos ayude tanto a ellos como a mí, a ver cuáles son los conocimientos esperados que debemos de reforzar; que no lo vean ellos, ¡ay! este acá saqué seis, cinco y este... pues es un número que sé que estoy mal; no... que ellos se fijen metas para ir colocando sus conocimientos un poquito más arriba; que les sirva como una herramienta para aumentar sus conocimientos y no como un número donde ellos etiquetan el nivel de aprendizaje que lograron.

Profesor I- Escenario 2: relacionado con compromiso con aprendizaje.

[Luego del proyecto] ya dimos un paso importante en cuanto a la revisión de cuentas, solo resta hacer que las cosas sucedan dando como resultado alumnas y alumnos con perfiles que los posibiliten a aprender a aprender y a aprender a convivir tal y como muestran nuestra misión y visión. 
EVALUAR EL DESEMPEÑO EN EL MARCO DEL MODELO EDUCATIVO BASADO EN COMPETENCIAS EN EDUCACIÓN SUPERIOR CON APOYO...

K. E. GALLARDO CÓRDOVA, M. ${ }^{\mathrm{a}}$ E. GIL RENDÓN Y A. L. GOVEA GARZA

Directivo I- Escenario 5: relacionado con evaluar para aprender.

Tenemos en cuenta que no todos los docentes se aplican con responsabilidad un plan de mejoramiento de retroalimentación para fortalecer los puntos débiles en el conocimiento y un gran compromiso que tenemos es de mejorar de... de hacer que todos los docentes, eh... estemos pensando hacía una meta definida y nos pongamos de acuerdo en cómo vamos a hacer, eh... el plan de mejoramiento.

\subsection{Mejora la comunicación en los procesos de evaluación}

Un segundo punto que también se aprecia como una constante en los planes de trabajo y en las declaraciones hechas en las entrevistas fue la intervención de los estudiantes de posgrado en abrir canales de comunicación para llevar el proceso de evaluación del aprendizaje a un nivel de entendimiento más elevado, tomando en consideración todos los puntos cardinales del proceso; es decir, hacia los directivos, los alumnos, entre profesores y con padres de familia.

Así, en esta categoría se identificó que en todos los escenarios, en especial en el escenario 5, se trabajó, reflexionó y se trazaron actividades de trabajo y acuerdos para ejercer más comunicación en los procesos de evaluación. De hecho, cabe destacar que los estudiantes de posgrado que realizaron las intervenciones no solo trabajaron en comunicar conocimientos contemporáneos sobre modelos y técnicas, también suscitaron el diálogo colegiado para la toma de acuerdos, difusión de resultados de aprendizaje a través de la retroalimentación y la comunicación con padres de familia, tanto en el tema del proceso como en el de entrega e interpretación de resultados. La Figura 4 muestra la relación de las subcategorías con la mejora de la comunicación en procesos de evaluación.

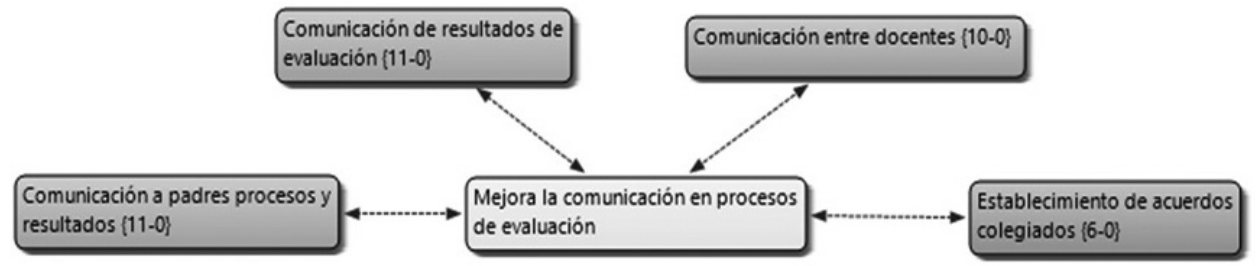

FiguRA 4: Red compuesta por las subcategorías que explican la mejora de comunicación en procesos de evaluación

Algunas de las más importantes declaraciones en torno a esta categoría son:

Directivo I- Escenario 3: relacionado con comunicación a padres: procesos y resultados.

Bueno, eh... notificar a los papas, ¿`sí?, de acuerdo a los consejos técnicos escolares que se han ido desarrollando tenemos que ponerles en conocimiento los resultados que se han ido obteniendo y bueno, en base a eso crean una mejora. ¿Cómo se va a crear esta mejora? La mejora se va a crear con la intervención de padres de familia de maestros, ¿sí?, de tutores y de directivos que prácticamente eso... eso ayudaría para... para poder salir adelante, eh... eh... en ese tipo de acción. 
Profesor 2 - Escenario 5: relacionado con establecimiento de acuerdos colegiados.

Bueno pues hay varias estrategias, Maritzabel, una es como ya decíamos mejorar los canales de comunicación, eh... también nos hemos propuesto hacer un banco de preguntas de manera que pues seamos coherentes con ese tipo de evaluación... básicamente eso es lo que hemos pensado hasta ahora.

\subsection{Mejora de habilidades docentes para evaluar}

Si bien es cierto que la intención de todos los planes de trabajo apuntaban a que las intervenciones incidirían precisamente en este rubro, en especial en el tema de retroalimentación, el seguimiento y diseño de instrumentos, esta categoría no se encontró en el primer lugar. De hecho, su frecuencia de aparición es un tanto baja en todos los escenarios, diferenciándose los escenarios I, 2 y 5 . Los aspectos a destacar son que las reflexiones giraron en torno al trabajo con nuevos conocimientos conceptuales y procedimentales que los docentes desconocían, mismos que permiten integrar y realizar de manera ordenada y sistemática los procesos de evaluación para dar seguimiento al desarrollo de los estudiantes. Estos nuevos saberes modificaron las propias concepciones sobre lo que es y significa evaluar el aprendizaje. También se reflexionó sobre el tiempo que demanda realizar bien los procesos. La Figura 5 contiene los elementos que integran esta categoría.

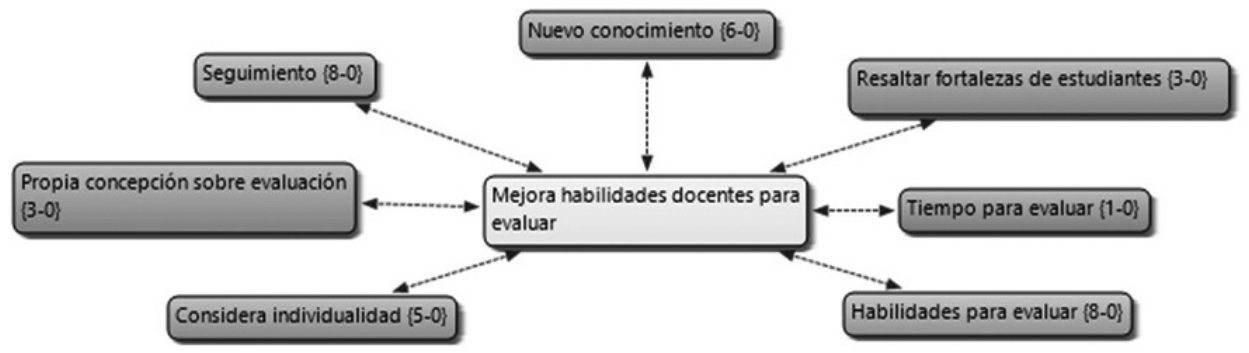

FIgURA 5: Red compuesta por las subcategorías que explican la mejora de las habilidades docentes para evaluar

Profesor 2- Escenario 2: relacionado con nuevo conocimiento.

[Yo]... no sabía que existía un modelo de retroalimentación que vinculaba muchos aspectos para hacer una evaluación integral. Después de que me explicaron el modelo y de revisar la primera guía que realizaron los niños, me di cuenta que faltaban muchos aspectos a evaluar, como escribir aspectos que fortalecieran elementos personales de cada niño y que mostraran motivación para las siguientes actividades.

Profesor 2- Escenario I: relacionado con nuevo conocimiento.

Ah porque aquí en la sede se trabaja con niños y niñas menores... se procura escribirles frases oraciones que... que los motive felicitándolos, ponerles caritas felices, sellitos... porque eso a los niños y a las niñas les agrada. Em... pero entonces eso da una 
EVALUAR EL DESEMPEÑO EN EL MARCO DEL MODELO EDUCATIVO BASADO EN COMPETENCIAS EN EDUCACIÓN SUPERIOR CON APOYO...

K. E. GALLARDO CÓRDOVA, M. ${ }^{a}$ E. GIL RENDÓN Y A. L. GOVEA GARZA

práctica empírica. Interesante saber que es parte de todo un modelo estructurado para hacer una buena retroalimentación. Entonces eso fue como los dos puntos interesantes, el fuerte trabajamos esa parte motivacional y el débil que no se trabajaba el feedforward* entonces ahí queda pues como... como ese compromiso para seguir trabajando.

[“Feedforward: elemento del modelo de evaluación de Hattie y Timperley (2007) que indica que la retroalimentación debe incorporar elementos sobre cómo la tarea actual y los procesos mentales que demanda servirán para enfrentar retos académicos posteriores].

Profesor 2- Escenario 7: relacionado con el seguimiento.

Este... pues el hecho a lo mejor que se requiere siempre incluir... de alguna manera estar monitoreando el trabajo de los alumnos con los tres tipos de evaluaciones. No dejarlos nunca a un lado y contemplar las tres para de alguna manera analizar y reflexionar sobre el trabajo de los alumnos.

\subsection{Innovación educativa para mejorar la evaluación}

En esta última categoría se encuentran vinculados aspectos relacionados con nuevos conocimientos y actividades que los profesores y directivos consideraron como innovaciones educativas. La más destacada fue justamente la inserción de un modelo de retroalimentación para orientar mejor los procesos de valoración y comunicación del aprendizaje alcanzado. Otro elemento en esta categoría es la distinción hecha entre evaluación sumativa y formativa, lo cual en algunos escenarios educativos, específicamente en el escenario 2, se propuso como algo a trabajar con mayor énfasis en los siguientes meses. Por último, también se hizo mención sobre la necesidad y trabajo en torno a la reconceptualización del modelo de evaluación de las instituciones de forma integral y no solo lo que le concierne a la evaluación formativa. La Figura 6 contiene los elementos vinculados con esta categoría.

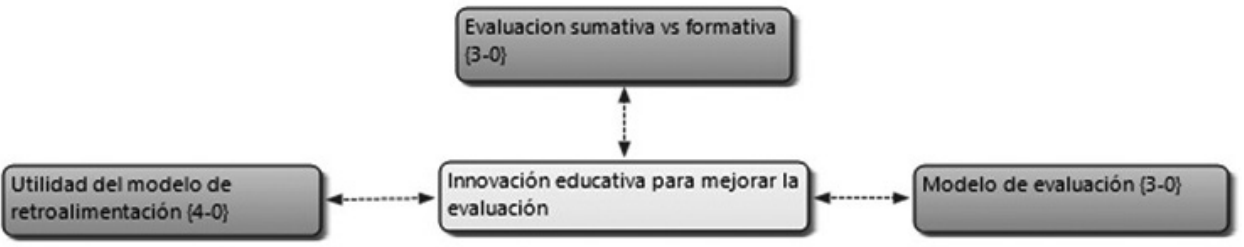

FiguRA 6: Red compuesta por las subcategorías que explican la innovación educativa para la mejora de la evaluación

Algunas declaraciones al respecto ilustran mejor los puntos anteriormente comentados.

Profesor I- Escenario i: relacionado con la utilidad del modelo de retroalimentación.

En esta segunda ocasión, pues se trató de mejorar, em... el modelo respecto a la primera implementación. Se encontró que el modelo es bastante interesante, en la medida que 
liga momentos, lo que se está retroalimentando o evaluando en el momento, en el presente se encadena a lo que se va a ver en el futuro. Entonces esa de parte de feedforward, fue uno de los elementos que más..., porque generalmente solamente se valoraba lo que los chicos y las chicas hacían con lo solicitado, pero nunca se le daba una retroalimentación en el cual se le fuera adelantando otras competencias u objetivos... contenidos que se fueran a tratar luego.

Profesor I- Escenario 2: relacionado con las mejoras en cuanto a evaluación sumativa y formativa y en general a la integración de nuevos elementos.

Bueno, uno de los retos más importantes se enfoca, eh... en otorgar la misma relevancia tanto la evaluación sumativa como la formativa. Involucrar a los padres de familia y alumnos en el proceso e incluir los estándares de ética, precisión, factibilidad y utilidad, así como un modelo de retroalimentación en los estudiantes con el fin de hacerlos participes.

Directivo I- Escenario 5: relacionado con modelo de evaluación.

Uno de los aspectos débiles en el proceso de evaluación es que la institución constantemente se está cambiando a los docentes. Y cada docente que llega tiene una visión diferente del proceso evaluativo. Tenemos en cuenta que no todos los docentes se aplican con responsabilidad un plan de mejoramiento de retroalimentación para fortalecer los puntos débiles en el conocimiento y un gran compromiso que tenemos es de mejorar de... de hacer que todos estemos pensando hacía una meta definida y nos pongamos de acuerdo en cómo vamos a hacer el plan de mejoramiento.

\section{Discusión y conclusiones}

A partir de los resultados, un primer aspecto a destacar es la posibilidad de apreciar congruencia entre las competencias estipuladas a priori y las declaraciones analizadas en torno al impacto de los proyectos de intervención (Gallardo Córdova, 2007; Lozano-Rodriguez y Herrera-Bernal, 2012). Una lección aprendida es que, si bien es cierto que las expectativas académicas en el curso de EA era que los alumnos incidieran fuertemente en el desarrollo de competencias docentes para mejorar los procesos de evaluación, no fue así. En verdad, fue inesperado pero igualmente apreciado que la reflexión más recurrente en los escenarios educativos fuera que el fin de la evaluación es el aprendizaje, idea que refleja una actitud y un cambio que podría privilegiar el trabajo más en procesos formativos que en sumativos. Este hallazgo también derivará en ajustes al diseño instruccional de EA, considerando el valor temático que cobra la evaluación formativa como consecuencia de este análisis.

Un segundo aspecto a destacar es la constante aparición de la competencia relacionada con detectar e impulsar el talento humano en cada una de las etapas de la evaluación del aprendizaje; tanto en poner a la población a ser evaluada en primer lugar, como en introducir procesos significativos de retroalimentación. El análisis llevó a determinar que en varios escenarios se abrieron canales de comunicación con los actores educativos, estimando mejoras también en las formas de presentar los resultados tanto a alumnos como a padres de familia (JCSE, 2OI2). 
EVALUAR EL DESEMPEÑO EN EL MARCO DEL MODELO EDUCATIVO BASADO EN COMPETENCIAS EN EDUCACIÓN SUPERIOR CON APOYO...

K. E. GALLARDO CÓRDOVA, M. ${ }^{a}$ E. GIL RENDÓN Y A. L. GOVEA GARZA

Por último, sobre la utilidad del uso de redes sociales para comprender mejor el contexto y el impacto del trabajo de los estudiantes, los resultados que dejan ver esta práctica permitió comprender cómo aspectos que se planean en el diseño cobran diversos significados en el ejercicio. Esta experiencia puede ser considerada una forma de aprovechar aún más la evaluación auténtica (Ashford-Rowe et al., 20r4; Monereo, (2007); tanto por parte de los estudiantes como también por parte de los responsables del diseño curricular e instruccional. Así, el uso de redes sociales puede ser conveniente en los siguientes aspectos: (I) propiciar una interacción académica apoyada en elementos contextuales que se exhiben en los escenarios educativos; (2) tomar una serie de evidencias en la práctica para estimar alcances, beneficios y también impactos del ejercicio de las capacidades; (3) contar con elementos que conlleven a la elaboración de mejores juicios para la evaluación; y (4) corroborar con más precisión qué tanto los estudiantes se están acercando a desarrollar lo que el perfil de egreso indica (Ministerio de Educación y Ciencia, 2007; Tobón, 2008).

Para futuros estudios, en el marco de la evaluación de desempeño con apoyo de redes sociales, es preciso seguir indagando en diferentes formas de interacción, de generación de evidencia para evaluar el ejercicio de las competencias y desarrollo de capacidades, así como la conformación de proyectos que vinculen de una forma más directa y cercana a las universidades con la comunidad, con proyectos que sean retadores y contribuyan al desarrollo social y educativo.

\section{Bibliografía}

Alarcón, J.; Hill, B. y Frites, C. (20I4) Educación basada en competencias: hacia una pedagogía sin dicotomías. Educ. Soc., Campindas, 35, 569-586. Obtenido de http://www.scielo. $\mathrm{br} / \mathrm{scielo}$. php?script=sci_arttextypid=SoIoI-733020140002000I3ylng=esynrm=iso.

Argudin, Y. (20Io) Educación basada en competencias. Nociones y antecedentes. México, D.F.: Trillas.

Ashror-Rowe, K.; Herrington, J. y Brown, C. (20I4) Establishing the critical elements that determine authentic assessment. Assessment \& Evaluation in Higher Education, 39 (2), 205222. Doi:I0.1080/02602938.2013.819566.

Boyd, D. y Ellison, N. (2008) Social Network Sites: Definition, History and Scholarship. Journal of Computer-Mediated Communication, I3, 210-230. Doi:I0.IIII/j.I083-6101.2007.00393.x.

Buzzetto-More, N. (20I4) An examination of undergraduate student's perceptions and predilections of the use of Youtube in the teaching and learning processs. Interdisciplinary Journal of E-Learning and Learning Objects, I0, 17-32. Obtenido de http://www.ijello.org/ Volumeio/IJELLOviopor7-032Buzzettoo437.pdf.

Coll, C. (2007) Las competencias en la educación escolar: algo más que una moda y mucho menos que un remedio. Obtenido de http://auladesecundaria.grao.com/revistas/aula/16i-lengua-y-expresion-plastica--las-competencias-en-la-educacion-escolar/las-competenciasen-la-educacion-escolar-algo-mas-que-una-moda-y-mucho-menos-que-un-remedio.

DeLors, J. et al. (1997) La educación encierra un tesoro: informe para la unesco de la Comisión Internacional sobre la Educación para el Siglo Veintiuno. Obtenido de http://disde. minedu.gob.pe/xmlui/bitstream/handle/123456789/1847/La\%2oeducacion\% 2oencierra\%20 un $\%$ 2otesoro.pdf?sequence $=$ I.

EARL, R. (2008) The Integration of Instructional Technology into Public Education: Promises and Challenges. E.T. Magazine, 42 (I), 5-13. Obtenido de http://isites.harvard.edu/fs/docs/ icb.topic87187.files/Earleo2.pdf. 
EVALUAR EL DESEMPEÑO EN EL MARCO DEL MODELO EDUCATIVO BASADO

EN COMPETENCIAS EN EDUCACIÓN SUPERIOR CON APOYO...

K. E. GALLARDO CÓRDOVA, M. ${ }^{\text {a }}$ E. GIL RENDÓN Y A. L. GOVEA GARZA

Evans, C. (20I4) Twitter for teaching: Can social media be used to enhance the process of learning? British Journal of Educational Technology, 45 (5), 902-915. Doi:Io.11I//bjet.12099.

Gallardo Córdova, K. (2007) Educación Basada en Competencias: Propuesta de un Modelo de Evaluación con Base en la Teoría de Expertos y Novatos y Aplicado al Aprendizaje de Excel. Disertación doctoral. Tecnológico de Monterrey. Monterrey, Nuevo León.

Gallardo Córdova, K. (2013) Evaluación del aprendizaje: retos y mejores prácticas. Monterrey, Nuevo León: Editorial Digital del Tecnológico de Monterrey. Obtenido de https:// www.editorialdigitaltec.com/index.php?route=product/productyproduct_id $=167$.

García, A. (2008) Las redes sociales como herramientas para el aprendizaje colaborativo: una experiencia con Facebook. Revista Re-Presentaciones Periodismo, Comunicación y Sociedad, 2 (5), 49-59. Obtenido de https://www.google.com.mx/webhp?sourceid=chrome-instantyion $=1$ yespv $=2$ yie $=U T F-8 \#$.

Gómez, M.; Roses, S. y Farias, P. (20I2) El uso académico de las redes sociales en universitarios. Comunicar, i9 (38), I3I-I38. Obtenido de http://www.redalyc.org/articulo.oa?id= 15823083016.

Gonzi, A. (200I) Análisis de las tendencias internacionales y los avances en educación y capacitación basadas en normas de competencia. En A. Argüelles Educación y capacitación basada en normas de competencia: Una perspectiva internacional (pp. 19-23). D.F., México: Limusa.

Hancock, D. (2007) Effects of performance assessment on the achievement and motivation of graduate students. Active learning in higher education, 8 (3), 219-231. Doi:Io.II77/I469787407081888.

Hattie, J. y Timperley, H. (2007) The power of feedback. Review of Educational Research, 77 (I), 8I-II2. Doi:IO.3102/003465430298487.

Ion, G. y CANO, C. (20II) La formación del profesorado universitario para la implementación de la evaluación por competencias. Educación XXI, I5 (2), 249-270. Obtenido de https:// www.google.com.mx/webhp? sourceid=chrome-instantyion=ryespv=2yie=UTF-8\#.

Joint Committee on Standards for Evaluation (20i2) Classroom assessment standards. Recuperado el is de febrero de 20I5, de http://www.jcsee.org/standards-development.

Lozano-Rodríguez, A. y Herrera-Bernal, A. (20I2) El enfoque educativo basado en competencias. Monterrey-Nuevo León, México: Editorial Digital del Tecnológico de Monterrey. Obtenido de https://www.editorialdigitaltec.com/index.php?route=product/produc.

Merriam, S. (2009) Qualitative research: A guide to design and implementation. San Francisco, CA. Jossey-Bass, Ed.

Ministerio de Educación y Ciencia (2007) El desarrollo de competencias docentes en la formación del profesorado. Madrid, España. Recuperado el I de marzo de 2015, de https://sede. educacion.gob.es/publiventa/vistaPrevia.action cod=I2281yarea $=$ E.

Monereo, C. (2007) La evaluación auténtica de competencias. En IV Congreso Regional de Educación. Competencias básicas y práctica educativa. Cantabria. Obtenido de https:// www.youtube.com/watch?v=tbugPzonMyk.

Morrow, J.; Moode, D.; Dicsh, J. y Kang, M. (20I6) Measurment and evaluation in human performance ( $\left.5 .^{\mathrm{a}} \mathrm{ed}.\right)$. Champaing, Illinois, Estados Unidos: Human Kinectics. Recuperado el I2 de febrero de 2016.

Panckhurst, R. y Marsh, D. (2OII) Utilización de redes sociales para la práctica pedagógica en la enseñanza superior impartida en Francia: perspectivas del educador y del estudiante. Revista de Universidad y Sociedad del Conocimiento (RUSC), 8 (I), 233-252. Obtenido de https://www.google.com.mx/webhp? sourceid=chrome-instantyion=ryespv=2yie $=U T F-8 \#$.

Pavié, I. (20II) Enfoque basado en competencias: orientaciones sobre sus procedimientos de evaluación. Revista Electrónica de Desarrollo de Competencias REDEC, 7 (I), II4-I47. Obtenido de http://redec.utalca.cl/index.php/redec/article/view/6r.

Ponce-Rojo, A.; Hernández- Vega, L.; Hernández-Contreras, J. y Fernández-RodríGUEZ, J. (20I2) Análisis de contenido de las interacciones en línea en cursos de pregrado 
EVALUAR EL DESEMPEÑO EN EL MARCO DEL MODELO EDUCATIVO BASADO EN COMPETENCIAS EN EDUCACIÓN SUPERIOR CON APOYO... K. E. GALLARDO CÓRDOVA, M. ${ }^{a}$ E. GIL RENDÓN Y A. L. GOVEA GARZA

usando Facebook en una modalidad de Blended Learning. Sinéctica, 39 (I), oI-I9. Obtenido de http://www.scielo.org.mx/scielo.php?script=sci_arttextypid=SI665-109X20I2000200006ylng=esytlng=es.

Proyecto Tuning América Latina (2007) Reflexiones y perspectivas de la educación superior en América Latina. Obtenido de http://www.scielo.org.co/scielo.php?script=sci_arttextypid=SI657-59972007000200oI2ylng=enynrm=iso $>$.

The Statistical Portal (s. f.) Obtenido de Social Network Users in Latin America: http:// www.statista.com/statistics/244929/number-of-social-network-users-in-latin-america/.

Товón, S. (2008) La formación basada en competencias en la educación superior: el enfoque complejo. Universidad Autónoma de Guadalajara. Obtenido de http://www.regioncadereyta.org /app/download/2623355/Formaci\% $3 \%$ B3n+basada+en+competencias.+Sergio+ Tob\% $\mathrm{C}_{3} \% \mathrm{~B}_{3}$ n.pdf.

Tovar-Gálvez, J.; García, G.; Cárdenas, N. y Fernández, Y. (20I2) Concepción, formación y evaluación por competencias: reflexiones en torno a posibles alternativas pedagógicas y didácticas. Educ. Soc., Campinas, 33 (I2I), I257-I273. Obtenido de http://www.cedes. unicamp.br.

TúÑEz, M. y Sixto, J. (20I2) Las redes sociales como entorno docente: análisis del uso de Facebook en la docencia universitaria. Revista de Medios y Educación, 4I, 77-92. Obtenido de http://www.redalyc.org/articulo.oa? $\mathrm{id}=36828247006$.

Valerio-Ureña, G.; Herrera-Murillo, D.; Villanueva-Puente, F.; Herrera-Murillo, N. y Rodríguez-Martínez, M. (2015) The Relationship between Post Formats and Digital Engagement: A Study of the Facebook Pages of Mexican Universities. Rusc. Universities and Knowledge Society Journal. RUSC, I2 (I). Doi:http://dx.doi.org/I0.7238/rusc.vi2ir.I887.

Valerio-Ureña, G.; Leyva, T.; Caraza, R. y Rodríguez- Martínez, M. (20I4) Redes sociales en línea y capacidad de memorización de los estudiantes universitarios. Revista Electrónica de Investigación Educativa, I6 (3), III8-I28. Obtenido de http://redie.uabc.mx/volI6no3/contenido-valerio-etal.html.

Venkatesh, V.; Croteau, A. y Rabah, J. (20I4) Perceptions of Effectiveness of Instructional Uses of Technology in Higher Education in an Era of Web 2.0. En 47th Hawaii International Conference on System Sciences (pp. IIo-II9). Doi:Io.IIog/HICSS.20I4.22

YIN, R. (2003) Case study research: Design and method (3. ${ }^{a}$ ed.). Thousands Oaks, CA, EE. UU.: Sage. 